\title{
Prognostic implications of aberrantly expressed methylation-driven genes in hepatocellular carcinoma: A study based on The Cancer Genome Atlas
}

\author{
JINZHONG LI $^{1}$, NING CHEN ${ }^{2}$ and XIAOBING GONG ${ }^{1}$ \\ Departments of ${ }^{1}$ Gastroenterology and ${ }^{2}$ General Medicine, The First Affiliated Hospital of Jinan University, \\ Guangzhou, Guangdong 510000, P.R. China
}

Received January 17, 2019; Accepted August 30, 2019

DOI: $10.3892 / \mathrm{mmr} .2019 .10771$

\begin{abstract}
RNA-Sequencing and methylation data for hepatocellular carcinoma (HCC) were downloaded from The Cancer Genome Atlas (TCGA). The aberrantly expressed methylation-driven genes in HCC and normal tissues were identified using the Limma package and the MethylMix algorithm. The Database for Annotation, Visualization and Integrated Discovery and ConsensusPathDB were used for Gene Ontology (GO) enrichment and pathway analysis. Univariate and multivariate Cox regression analyses were used to construct a prognostic risk model of HCC. Survival curve and receiver operating characteristic (ROC) curves were applied to evaluate the clinical utility of the risk model. A total of 238 methylation-driven genes were successfully identified from cancer and normal tissues. GO enrichment analysis indicated that these genes functioned in the extracellular space, interfering with lipid metabolism in hepatocytes and regulating adaptive immune responses. In total, 14 relevant pathways were identified. The following prognostic risk model was generated: Risk score $=$ CALML3 (degree of methylation) $\mathrm{x}(-4.860)+\mathrm{CCNI} 2 \mathrm{x}(2.071)+$ TNFRSF12A $\mathrm{x}(-3.369)+$ IFITM1 $x(1.203)+$ ENPP7P13 x (-1.366) + DDT x (2.139) + RASAL2-AS1 x (-1.384) + ANKRD22 x (-3.215). The median risk score $(0.970)$ derived from this model was set as cutoff
\end{abstract}

Correspondence to: Dr Xiaobing Gong, Department of Gastroenterology, The First Affiliated Hospital of Jinan University, 601 West Huangpu Avenue, Guangzhou, Guangdong 510000, P.R. China

E-mail: gongxb3450@hotmail.com

Abbreviations: BP, biology process; CC, cellular component; DAVID, The Database for Annotation, Visualization and Integrated Discovery; GO, Gene Ontology; HCC, hepatocellular carcinoma; IGF, insulin-like growth factor; IGFBPs, insulin-like growth factor binding proteins; $\mathrm{MF}$, molecular function

Key words: The Cancer Genome Atlas, hepatocellular carcinoma, methylation, biomarkers, Cox proportional hazards regression, survival analysis, prognosis value for assigning patients to high- or low-risk group. The 5 -year survival rate was $35.8 \%$ [95\% confidence interval $(\mathrm{CI})=27.1-47.4 \%]$ in the high-risk group and $61.7 \%(95 \%$ $\mathrm{CI}=51.4-74.2 \%)$ in the low-risk group $(\mathrm{P}<0.0001)$. The ROC curve showed an area under the curve of 0.742 , indicating that this model is appropriate for predicting the survival rate of patients. Furthermore, the methylation and expression levels of two key genes, tumor necrosis factor superfamily member $12 \mathrm{~A}$ and D-dopachrome decarboxylase, were significantly associated with prognosis and were correlated with cg00510447, cg26808293, cg11060661 and cg16132339 methylation. In conclusion, a prognostic risk model for HCC is proposed based on the bioinformatic analysis of methylation-driven genes. The findings of the present study may improve understanding of the pathogenesis and prognosis of HCC.

\section{Introduction}

Primary liver cancer is the second leading cause of cancer-related mortality in men globally and the sixth leading cause of cancer-related mortality in women (1). According to the statistics from the International Agency for Research on Cancer of the World Health Organization, there were $>782,000$ cases of primary liver cancer in 2012, making liver cancer the fifth and ninth most common cancer among men and women, respectively (2). Most risk factors for liver cancer, such as alcohol, are commoner in men than in women, so the prevalence of hepatocellular carcinoma (HCC) in men is 2-3 times higher than in women (1).

Cell characteristics are under control of and maintained by both genetic and epigenetic mechanisms (3). Epigenetics is defined as inheritable changes in gene expression with no alterations in DNA sequence (4). Methylation, the most important and common epigenetic modification, is an important means of regulating genome function (5). Decreases in the genome-wide methylation level is an important indicator of early cancer and may be significantly correlated with the severity and metastasis of cancer (6). The main reason underlying this phenomenon is the demethylation of repeat sequences, which leads to genome instability $(7,8)$. Aberrant hypermethylation of $\mathrm{CpG}$ islands in specific promoter regions is another important phenomenon in the late stage of cancer, 
leading to changes in chromosomal structure, silencing of tumor suppressor genes and other cancer-related genes, thereby promoting cancer cells to adapt to the microenvironment and metastasis $(9,10)$. Previous studies have been conducted to examine the implication of methylation in the diagnosis, treatment response and prognosis of various malignancies, such as lung (11), breast (12), and ovarian cancer (13). It has also been reported that coactivator-associated arginine methyltransferase 1-mediated GAPDH methylation is a key regulatory mechanism of glucose metabolism in liver cancer (14). In addition, it has also been found that the mRNA expression of tumor suppressor P14ARF is regulated by DNA methylation in primary liver cancer; DNA methylation of the P14ARF gene may be related to the occurrence and tumor-node-metastasis stage of primary liver cancer (15).

The Cancer Genome Atlas (TCGA) (16), a public database used in bioinformatics analysis, has complete patient data and is conducive to the analysis of cancer progression and prognosis. In the present study, HCC-related methylation-driven genes were identified from TCGA database, and the correlation between the identified genes and HCC progression and prognosis was analyzed. A series of bioinformatic and survival analyses were conducted to identify the biomarkers associated with methylation and constructed a prognostic risk model for predicting the prognosis of patients with HCC.

\section{Materials and methods}

Study population and data processing. The HCC-related level 3 RNA-sequencing (Seq) data and methylation data were downloaded from TCGA (https://gdc.cancer.gov/). Specifically, the Perl programming language (http://www. perl.org/) was used to extract matrix files from the RNA-seq and methylation data files. There were 122 women and 255 men; 204 patients were aged $\geq 60$ and 172 patients aged $<60$. The clinical data of the patients with HCC are summarized in Table I. The RNA-Seq and methylation matrix files were merged using the Limma package (17) and the MethylMix algorithm. Following the merge, the genes meeting all of the following three conditions were defined as methylation-driven genes: i) Gene expression levels differ between normal and cancer tissues; ii) methylation levels differ between normal and cancer tissues; and iii) the degree of DNA methylation is correlated with gene expression. The Pheatmap package (1.0.12, https://CRAN.R-project. org/package $=$ pheatmap) was used to cluster the methylation-driven genes.

Gene Ontology (GO) and pathway enrichment analysis of aberrantly expressed methylation-driven genes. The Database for Annotation, Visualization and Integrated Discovery (DAVID 6.7; https://david.ncifcrf.gov/) was used to perform GO enrichment analysis of the aberrant methylation-driven genes. A list of differentially expressed methylation-driven genes was submitted to the functional annotations tool in DAVID (screening criteria, $\mathrm{P}<0.01$ ).

Differentially expressed methylation-driven genes were analyzed using the overexpression analysis function of ConsensusPathDB 3.4.1 (http://cpdb.molgen.mpg.de/). The Kyoto Encyclopedia of Genes and Genomes database
Table I. Summary of the clinical data for hepatocellular carcinoma from The Cancer Genome Atlas.

\begin{tabular}{lc}
\hline Characteristics & No. of samples, $\mathrm{n}(\%)$ \\
\hline Age, years & $32(8.5)$ \\
$\leq 50$ & $342(91.5)$ \\
$>50$ & \\
Sex & $122(32.4)$ \\
Female & $255(67.6)$ \\
Male & \\
Stage & $175(49.6)$ \\
I & $87(24.6)$ \\
II & $86(24.4)$ \\
III & $5(1.4)$ \\
IV & \\
Survival state & $245(65.0)$ \\
Alive & $132(35.0)$ \\
Deceased & \\
Race & $19(5.2)$ \\
American & $161(43.9)$ \\
Asian & $187(50.9)$ \\
Caucasian & \\
\hline
\end{tabular}

(conducted in ConsensusPathDB 3.4.1) was used to perform pathway enrichment analysis for the differentially expressed methylation-driven genes taking $\mathrm{P}<0.01$ as a cutoff value.

Screening for prognosis-related signatures and risk score calculations. The univariate Cox regression model was used to calculate the association between the methylation level of each aberrant methylation-driven gene and the overall survival (OS) of the patient. Genes were considered statistically significant in univariate Cox analysis when $\mathrm{P}<0.05$. The amount of $\mathrm{Z}$-value represents the distance between the original score and the parent mean, measured in standard deviation. When the original score is lower than the average, $\mathrm{Z}$ is negative and vice versa. Multivariate Cox regression was used in the R language (3.5.1, https://www.r-project. org/) Survival Package (2.44-1.1, https://CRAN.R-project. org/package $=$ survival) to screen the independent prognostic ability of the aberrant methylation-driven genes (18). A prognostic risk assessment model was constructed by integrating the information of the aberrant methylation-driven genes.

The log-rank test was used to calculate P-values and the prognostic correlation coefficient $\beta$. The risk score was defined as follows: Risk score $=\beta_{\text {gene1 }} \times$ methyl $_{\text {gene } 1}+\beta_{\text {gene2 }} \times$ methylgene2 $+\cdots \cdot+\beta_{\text {gene (n) }} \times$ methyl $_{\text {gene (n) }}$, where $\beta$ is the prognostic correlation coefficient and methyl is the methylation level of the corresponding gene. The risk score of each sample was calculated and the median risk score was set as the cutoff value for assigning the samples into the low- and high-risk groups $(11,19)$. The Kaplan-Meier method was used to plot the survival curves for the low- and high-risk groups. Differences between groups were evaluated using the log-rank test. Receiver operating characteristic (ROC) curves were drawn to 


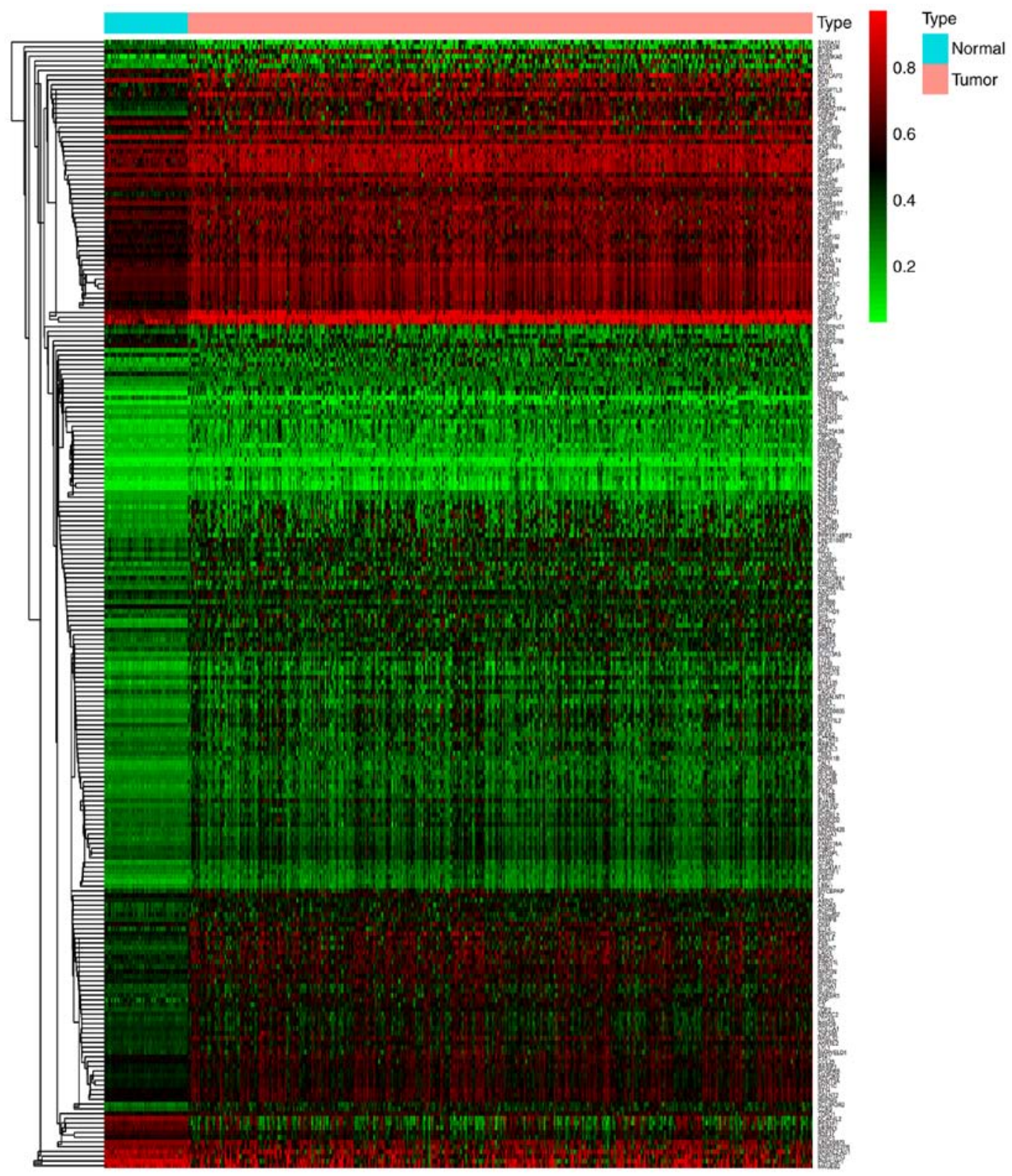

Figure 1. Heat map of the 238 methylation-driven genes. The Pheatmap R package was used for bidirectional hierarchical clustering of differentially expressed methylation-driven genes in hepatocellular carcinoma and adjacent tissues. Red indicates hypermethylation of the gene in the sample and green indicates hypomethylation of the gene.

predict the survival time of patients in terms of the degree of gene methylation.

Survival and correlation analysis. Survival analysis was performed by combining the identified prognostic aberrantly expressed methylation-driven genes with the corresponding gene expression data. The survival curves were rendered using the Survival Package in $\mathrm{R}$. The resulting prognostic genes were considered as key genes in HCC because their methylation degree and gene expression level were significantly correlated with prognosis. The abnormal methylation was thought to be associated with gene expression. In order to examine correlation, methylation related loci of the key genes were extracted from the downloaded HCC methylation data to assess the correlation between the key gene methylation loci and gene expression (the screening criteria were |Corl. P $>|0.3|$; Cor is correlation).

\section{Results}

TCGA data processing and screening of differentially expressed methylation-driven genes. HCC-related level 3 RNA-Seq data (374 HCC samples and 50 control samples) and methylation data (380 HCC samples and 50 control samples) were downloaded from TCGA. A total of 238 methylation-driven genes were identified according to the predefined conditions using Perl script and the $\mathrm{R}$ package. The Pheatmap package was used to cluster the methylation-driven genes (Fig. 1). The distribution map of the degree of methylation of some of the differentially methylated genes is shown in Fig. 2.

GO enrichment and pathway analysis of aberrantly expressed methylation-driven genes. Functional enrichment analysis was conducted on the identified genes to understand the functional role of the aberrant methylation-driven genes in 
A

Mixture model of CALML3
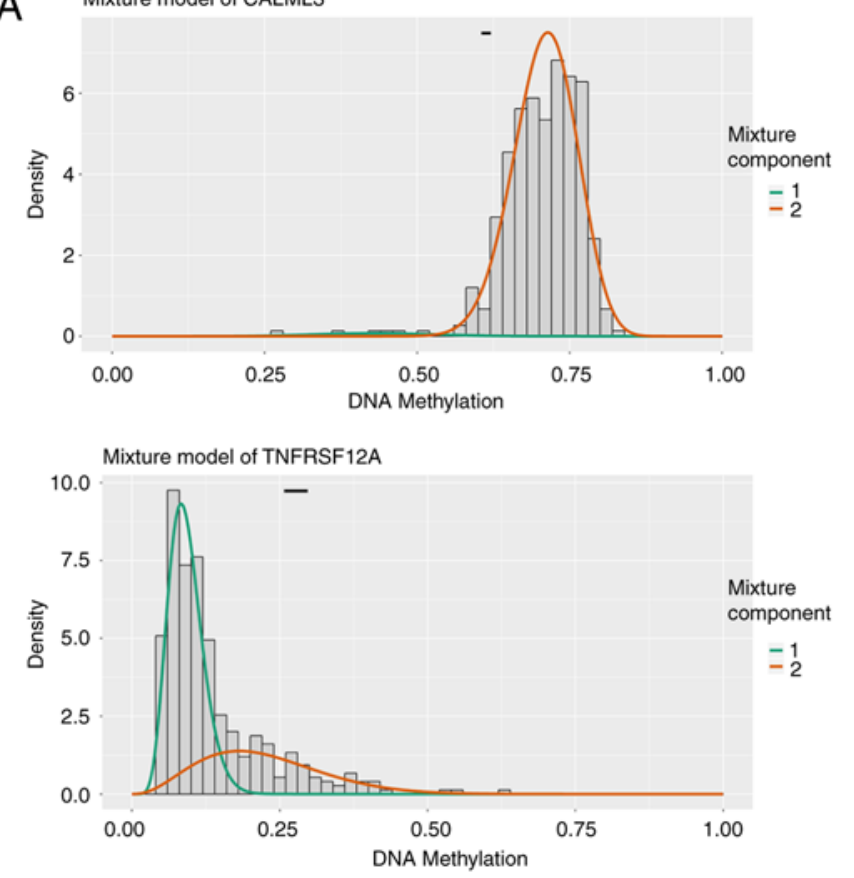

$\mathrm{B}$

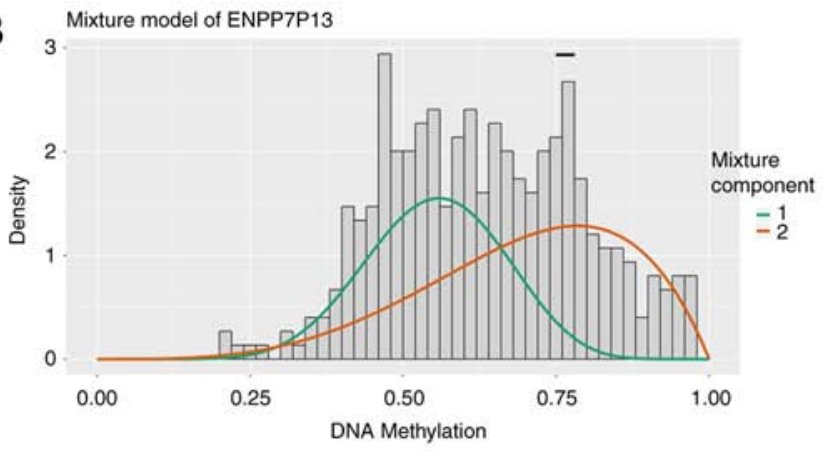

Mixture model of RASAL2-AS1

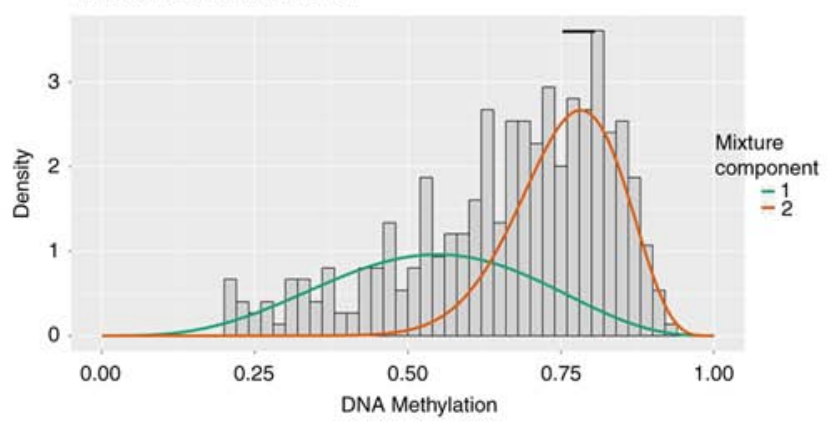

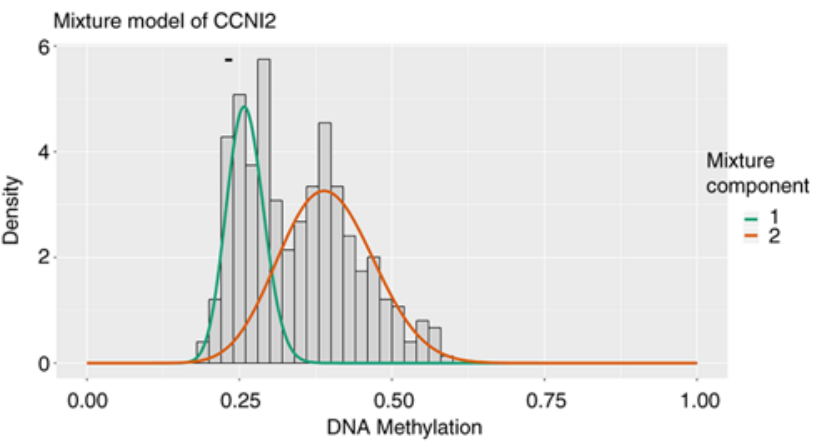

Mixture model of IFITM1
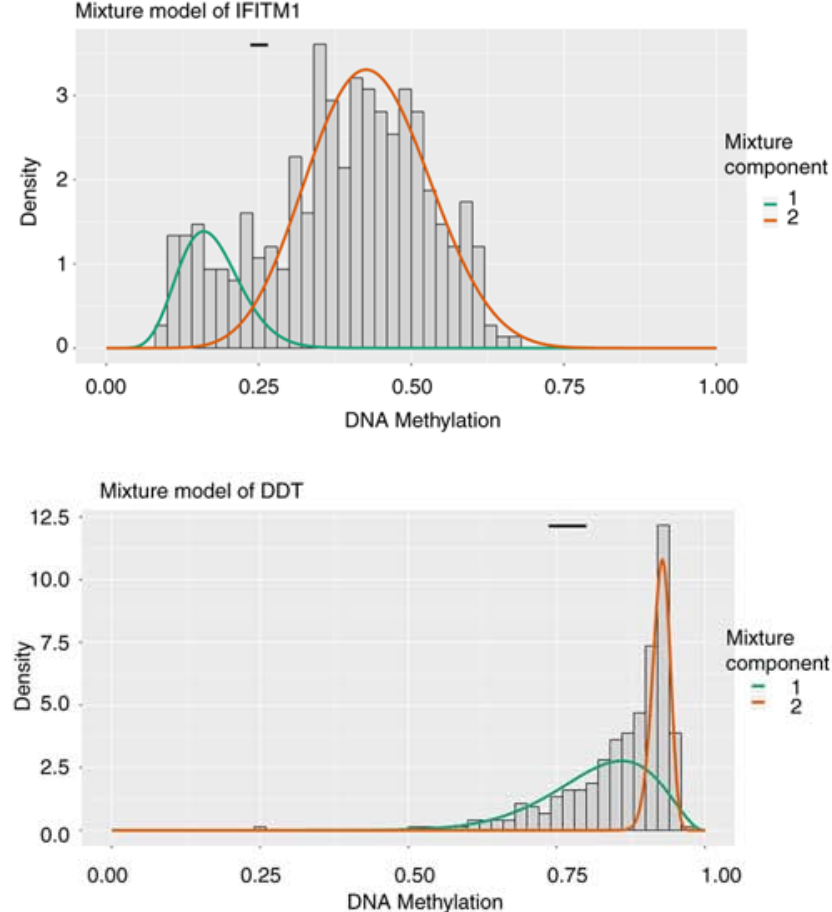

Mixture model of ANKRD22

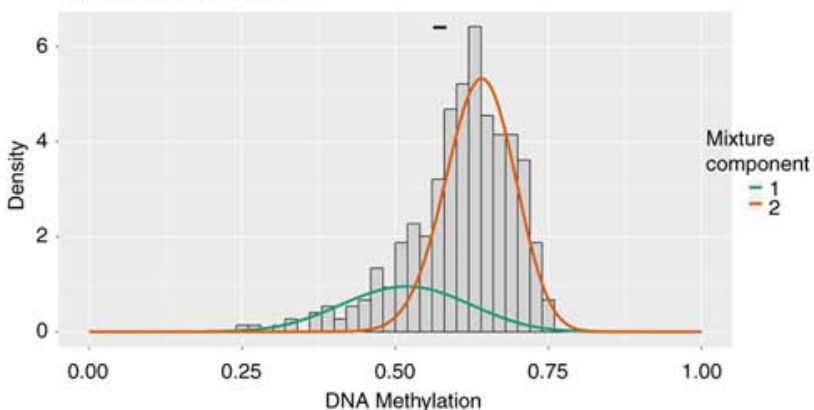

Figure 2. Histogram showing the distribution of the degree of methylation of eight differentially expressed methylation-driven genes. (A and B) The abscissa indicates the degree of methylation and the ordinate represents the number of methylated samples. The histograms display the distribution of methylation levels and the red and green curves are both the simulated trend curve of the methylation distribution of cancer group. The mixture component represents the methylation subgroup distribution. The black horizontal line above the graphs indicate the methylation level distribution of the normal sample. The distribution of methylation is different between the cancer and normal samples. In parts The greater the distance between the cancer and the normal samples, the greater the difference in methylation levels. CALM3, calmodulin-like protein 3; CCNI2, cyclin I family member 2; TNFRSF12A, tumor necrosis factor receptor superfamily member 12A; IFITM1, interferon induced transmembrane protein 1; ENPP7P13, ectonucleotide pyrophosphatase/phosphodiesterase 7 pseudogene 13; DDT, D-dopachrome decarboxylase; RASAL2-AS1, RASAL2-antisense RNA1; ANKRD22, ankyrin repeat domain 22.

HCC. The results showed that the aberrantly expressed methylation-driven genes were associated with molecular functions (MFs), biological processes (BPs) and cellular components (CCs). In the BP cluster, the genes were mainly associated with 'epithelial cell morphogenesis in placental branching', 'positive regulation of lipid catabolism', 'cholesterol homeostasis', 'cholesterol metabolic process' and 'lipoprotein metabolic process'. The MF included 'endopeptidase inhibitor activity', 'cholesterol transporter activity' and 'transcription factor activity', 'sequence-specific DNA binding'. The CC cluster included 'extracellular space', 'blood microparticles' and the 'apical plasma membrane' (Table II). 
Table II. GO functional enrichment of aberrantly expressed methylation-driven genes.

\begin{tabular}{|c|c|c|c|c|c|}
\hline Function & Term & Enrichment & Count & P-value & FDR \\
\hline \multirow[t]{6}{*}{$\mathrm{BP}$} & GO:0060672 & Epithelial cell morphogenesis in placental branching & 3 & 0.00036 & 0.581 \\
\hline & GO:0050996 & Positive regulation of lipid catabolic process & 3 & 0.00118 & 1.897 \\
\hline & GO:0002819 & Regulation of adaptive immune response & 3 & 0.00176 & 2.813 \\
\hline & GO:0042632 & Cholesterol homeostasis & 5 & 0.00542 & 8.427 \\
\hline & GO:0008203 & Cholesterol metabolic process & 5 & 0.00671 & 10.339 \\
\hline & GO:0042157 & Lipoprotein metabolic process & 4 & 0.00836 & 12.724 \\
\hline \multirow[t]{4}{*}{$\mathrm{CC}$} & GO:0072562 & Blood microparticle & 8 & 0.00146 & 1.822 \\
\hline & GO:0005615 & Extracellular space & 26 & 0.00682 & 8.244 \\
\hline & GO:0016324 & Apical plasma membrane & 9 & 0.0152 & 17.546 \\
\hline & GO:0005576 & Extracellular region & 28 & 0.0176 & 19.967 \\
\hline \multirow[t]{3}{*}{ MF } & GO:0004866 & Endopeptidase inhibitor activity & 5 & 0.00106 & 1.438 \\
\hline & GO:0017127 & Cholesterol transporter activity & 3 & 0.0121 & 15.423 \\
\hline & GO:0003700 & Transcription factor activity, Sequence-specific DNA binding & 20 & 0.0128 & 16.189 \\
\hline
\end{tabular}

GO, gene ontology; FDR, false discovery rate; $\mathrm{BP}$, biological processes; $\mathrm{CC}$, cell components; MF, molecular function.

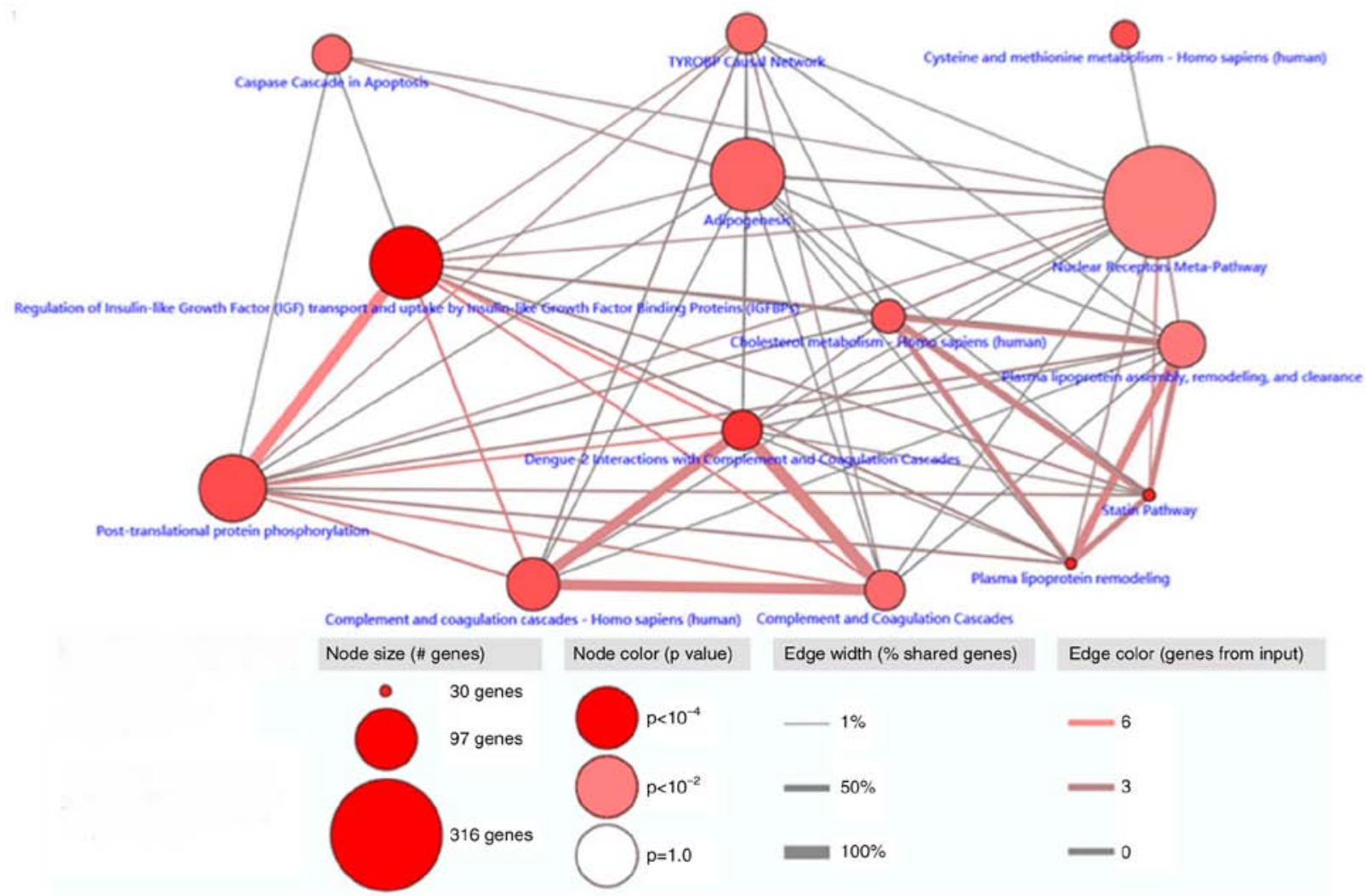

Figure 3. Pathway analysis of the differentially expressed methylation-driven genes. The nodes represent pathways and the lines denote the relationship between the pathways. The node size indicates the number of genes involved in the pathway, with a larger number indicating more genes are involved in the pathway. The node color represents the significance of enrichment, with darker red representing a higher degree of enrichment. The edge width represents the percentage of shared genes and the edge color indicates the number of input of genes.

Pathway enrichment analysis was performed on the 238 differentially expressed methylation-driven genes using ConsensusPathDB. A total of 14 pathways with significance $(\mathrm{P}<0.01)$ were identified, the most relevant of which was the pathway responsible for the regulation of insulin-like growth factor (IGF) transport and uptake by insulin-like growth factor binding proteins (IGFBPs), plasma lipoprotein remodeling and statins (Fig. 3).

Prognostic implications of aberrantly expressed methylation-driven genes. Univariate Cox regression analysis of the aberrantly expressed methylation-driven genes in patients 
Table III. Methylation-driven genes significantly associated with overall survival in hepatocellular carcinoma using univariate Cox regression analysis.

\begin{tabular}{lrrr}
\hline Gene & \multicolumn{1}{c}{ HR } & $\mathrm{Z}$ & P-value \\
\hline ANKRD22 & 0.033 & -3.352 & 0.001 \\
LIME1 & 7.012 & 3.181 & 0.001 \\
RASAL2-AS1 & 0.187 & -3.086 & 0.002 \\
LINC00346 & 0.064 & -3.054 & 0.002 \\
ENPP7P13 & 0.164 & -2.971 & 0.003 \\
TNFRSF12A & 0.050 & -2.944 & 0.003 \\
GPR75 & 6.951 & 2.830 & 0.005 \\
DDT & 24.444 & 2.442 & 0.015 \\
PABPC1P4 & 6.664 & 2.441 & 0.015 \\
EPHX3 & 5.015 & 2.379 & 0.017 \\
SFN & 0.248 & -2.352 & 0.019 \\
TMPRSS5 & 0.059 & -2.343 & 0.019 \\
RABGGTB & 0.235 & -2.249 & 0.024 \\
FITM1 & 10.908 & 2.242 & 0.025 \\
CAPS & 0.260 & -2.199 & 0.028 \\
IFITM1 & 4.161 & 2.179 & 0.029 \\
CCNI2 & 7.200 & 2.152 & 0.031 \\
RPL39L & 6.896 & 2.148 & 0.032 \\
USP44 & 4.258 & 2.138 & 0.033 \\
LCAT & 9.746 & 2.130 & 0.033 \\
RARRES2P8 & 0.311 & -2.069 & 0.039 \\
HPX & 6.227 & 2.057 & 0.040 \\
NQO1 & 0.398 & -2.055 & 0.040 \\
GSTM1 & 3.443 & 2.047 & 0.041 \\
RNF135 & 3.878 & 2.030 & 0.042 \\
PPIEL & 6.030 & 2.030 & 0.042 \\
LDHB & 3.933 & 1.998 & 0.046 \\
CALML3 & 0.067 & -1.971 & 0.049 \\
\hline NR & &
\end{tabular}

HR, hazard ratio.

with HCC showed that 28 genes were significantly associated with OS $(\mathrm{P}<0.05$; Table III). Multivariate Cox regression analysis was used to construct a prognostic risk model. As shown in Table IV, eight genes were found with prognostic significance: Calmodulin-like protein 3 (CALML3), cyclin I family member 2 (CCNI2), tumor necrosis factor receptor superfamily member 12A (TNFRSF12A), interferon induced transmembrane protein 1 (IFITM1), ectonucleotide pyrophosphatase/phosphodiesterase 7 pseudogene 13 (ENPP7P13), D-dopachrome decarboxylase (DDT), RASAL2-antisense RNA1 (RASAL2-AS1) and ankyrin repeat domain 22 (ANKRD22). The following risk model was constructed: Risk score $=$ CALML3 (degree of methylation) $x(-4.860)+$ CCNI2 $\mathrm{x}(2.071)+$ TNFRSF12A x (-3.369) + IFITM1 x (1.203) + ENPP7P13 x (-1.366) + DDT x (2.139) + RASAL2-AS1 x $(-1.384)+$ ANKRD22 $\times(-3.215)$. The median risk score derived from this model was set as the cutoff value for stratifying the 376 patients into the high-risk $(n=188)$ or low-risk groups $(n=188)$. The relationship between HCC risk and the degree
Table IV. Methylation-driven genes significantly associated with overall survival in hepatocellular carcinoma using multivariate Cox regression analysis.

\begin{tabular}{lrrrrl}
\hline Gene & coef & exp(coef) & se(coef) & Z-value & P-value \\
\hline CALML3 & -4.860 & 0.008 & 1.344 & -3.617 & 0.0003 \\
CCNI2 & 2.071 & 7.931 & 1.095 & 1.891 & 0.059 \\
TNFRSF12A & -3.369 & 0.034 & 1.008 & -3.342 & 0.0008 \\
IFITM1 & 1.203 & 3.329 & 0.716 & 1.680 & 0.093 \\
ENPP7P13 & -1.366 & 0.255 & 0.711 & -1.920 & 0.055 \\
DDT & 2.139 & 8.493 & 1.351 & 1.584 & 0.113 \\
RASAL2-AS1 & -1.384 & 0.251 & 0.570 & -2.426 & 0.015 \\
ANKRD22 & -3.215 & 0.040 & 1.060 & -3.033 & 0.002 \\
\hline
\end{tabular}

coef, coefficient; exp (coef), HR-hazard ratio; se, standard error; Z-value, Wald statistic, $\mathrm{z}=\mathrm{coef} / \mathrm{se}$ (coef); CALM3, calmodulin-like protein 3; CCNI2, cyclin I family member 2; TNFRSF12A, tumor necrosis factor receptor superfamily member 12A; IFITM1, interferon induced transmembrane protein 1; ENPP7P13, ectonucleotide pyrophosphatase/phosphodiesterase 7 pseudogene 13; DDT, D-dopachrome decarboxylase; RASAL2-AS1, RASAL2-antisense RNA1; ANKRD22, ankyrin repeat domain 22.

of methylation was illustrated in Fig. 4. The Kaplan-Meier method was used to plot the survival curves for the low- and high-risk HCC groups (Fig. 5). The 5-year survival rate of patients was $35.8 \%$ [95\% confidence interval $(\mathrm{CI})=27.1-47.4 \%$ ] in the high-risk group and $61.7 \%(95 \% \mathrm{CI}=51.4-74.2 \%)$ in the low-risk group. The difference between the groups was significant $(\mathrm{P}<0.0001)$. The ROC curve had an area under the curve of 0.742 , indicating that this model is able to predict the survival rate of patients with $\mathrm{HCC}$.

Association between different methylation loci and gene expression. $\mathrm{P}<0.05$ was used as a screening standard for combinatorial survival. The methylation degree and gene expression level of TNFRSF12A and DDT were significantly associated with the prognosis of HCC (Fig. 6). In addition, the methylation loci of the prognostic genes were identified using TCGA. The correlation between the different methylation loci and gene expression was analyzed using |Corl. P $>|0.3|$ was used as the screening cutoff value. It was found that the expression of TNFRSF12A and DDT was related to the methylation level of multiple loci (Fig. 7). TNFRSF12A was generally in a hypomethylated high expression state in patients with HCC, while DDT was in a hypermethylated low expression state, suggesting that TNFRSF12A may be a proto-oncogene and DDT may be a tumor suppressor gene.

\section{Discussion}

The incidence of hepatitis B (HBV) related $\mathrm{HCC}$ is expected to decrease with the increasing rate of vaccinations against HBV and the cumulative impact of new generation antiviral drugs (20). However, alcoholism, diabetes, obesity and metabolic syndrome play an important role in hepatocellular carcinoma in regions with a low prevalence of $\mathrm{HBV}$, 


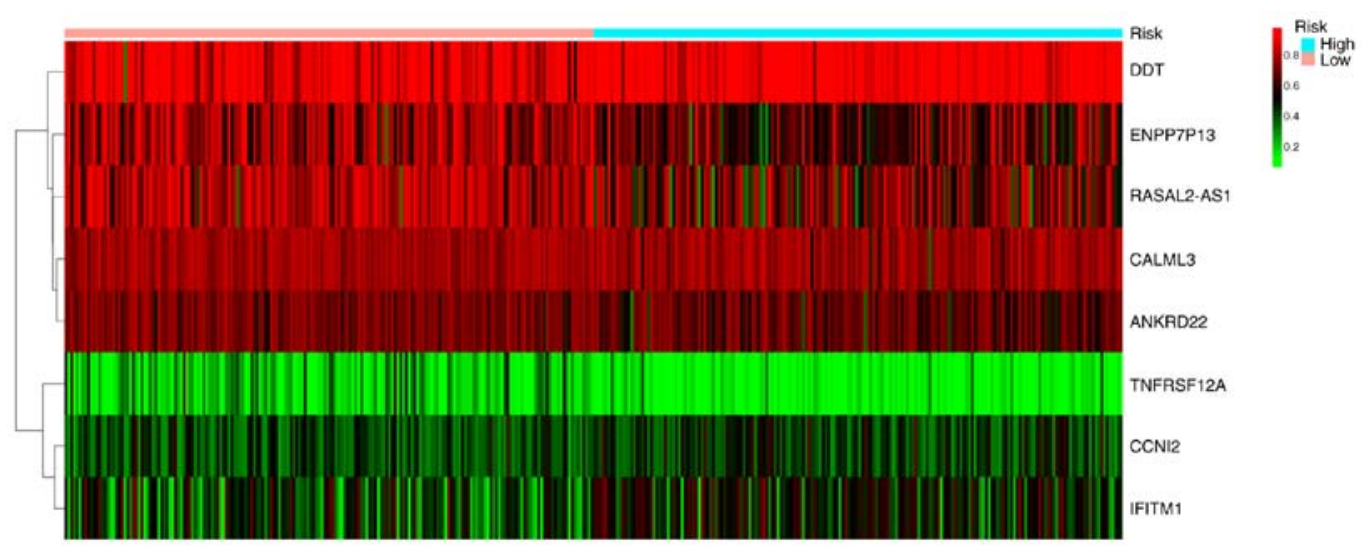

Figure 4. Analysis of the methylation heat map in high- and low-risk groups of liver cancer. The Pheatmap R package was used for the bidirectional hierarchical clustering of prognostic genes in high- and low-risk groups. Pink represents the low-risk group and blue represents the high-risk. Red indicates hypermethylation of the gene in the sample, while green represents hypomethylation. CALM3, calmodulin-like protein 3; CCNI2, cyclin I family member 2; TNFRSF12A, tumor necrosis factor receptor superfamily member 12A; IFITM1, interferon induced transmembrane protein 1; ENPP7P13, ectonucleotide pyrophosphatase/phosphodiesterase 7 pseudogene 13; DDT, D-dopachrome decarboxylase; RASAL2-AS1, RASAL2-antisense RNA1; ANKRD22, ankyrin repeat domain 22 .
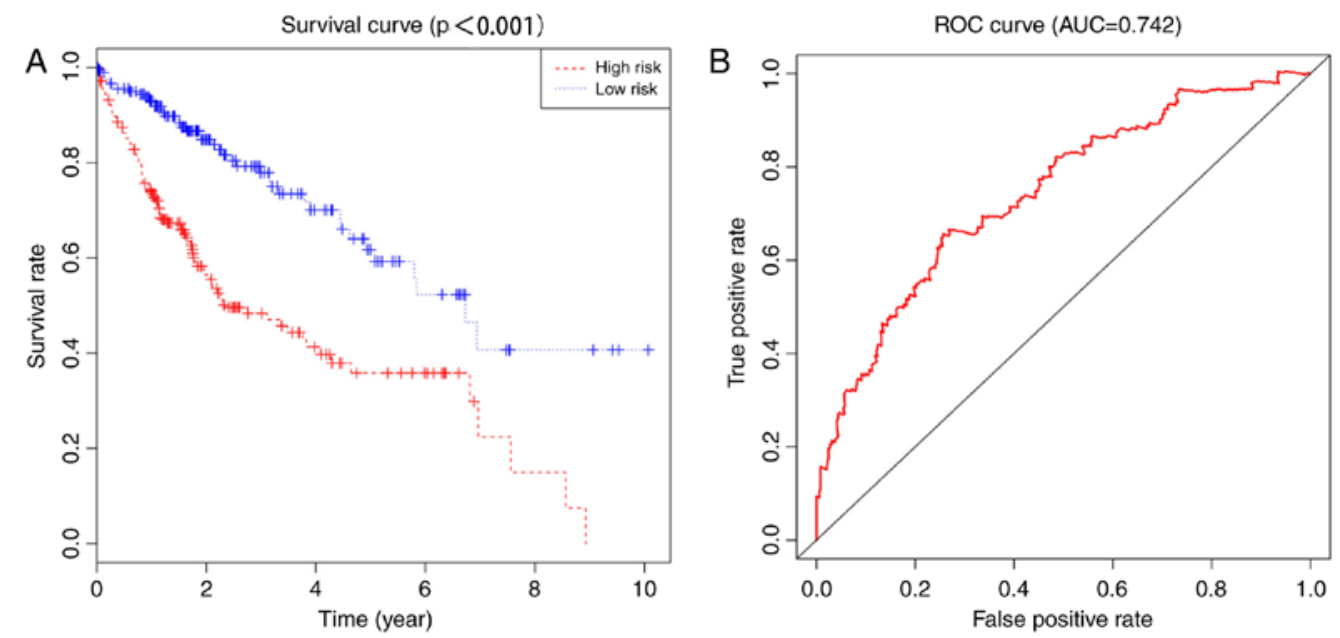

Figure 5. Survival and ROC curve analysis of the prognostic model. (A) Kaplan-Meier survival curves showing the overall survival based on the risk cutoff value. (B) Time-dependent ROC curve analysis of the 5-year survival prediction using the eight genes identified. ROC, Receiver operating characteristic; AUC, area under the curve.

especially in western populations. As a result, the prevalence of HCC will continue to increase (21). Diet is also a primary factors affecting DNA methylation (22). DNA methylation is dependent on the availability of s-adenosylmethionine (SAM), while methyl donors in food (including folate, betaine and choline) are linked to the synthesis of SAM $(23,24)$. A methyl-deficient diet has been reported to reduce the concentration of SAM in the liver, leading to the methylation of $\mathrm{CpG}$ islands in 164 genes in the livers of mice; these genes are involved in DNA damage and repair, lipid and glucose metabolism, and the progression of fibrosis, which can ultimately lead to $\operatorname{HCC}(25,26)$.

At present, the pathogenesis of HCC has not been clearly elucidated. HCC is related with multiple gene mutations and epigenetic aberrations (27). Mechanistically, DNA methylation leads to transcriptional silencing in one of two ways: i) Methylation at the $\mathrm{CpG}$ site hinders the spatial accessibility of transcription factors to homologous binding sites in various gene promoters (28); and ii) the direct binding of methyl-CpG-binding domain proteins to methylated DNA, causing transcriptional inhibition (29). It was hypothesized that the methylation-driven genes aberrantly expressed between patient with HCC and normal samples may be useful for predicting the prognosis of HCC. To test this, R script was used to combine methylation and transcriptome data, identifying 238 methylation driven-genes. The subsequent functional enrichment of these genes revealed the potential functional targets, such as 'blood microparticles', 'extracellular space' and 'apical plasma membranes', interfering with lipid metabolism in hepatocytes and the 'regulation of adaptive immune responses' by affecting 'endopeptidase inhibitor activity', 'cholesterol transporter activity', 'transcription factor activity', 'sequence-specific DNA binding'. The resulting pathways predominantly regulated IGF transport and IGFBP uptake, plasma lipoprotein remodeling and statins. These results suggested that high-fat, hypomethylated diets can affect DNA methylation, further affecting lipid metabolism in the body. This cycle can lead to liver steatosis, inflammation, fibrosis and cancer. 

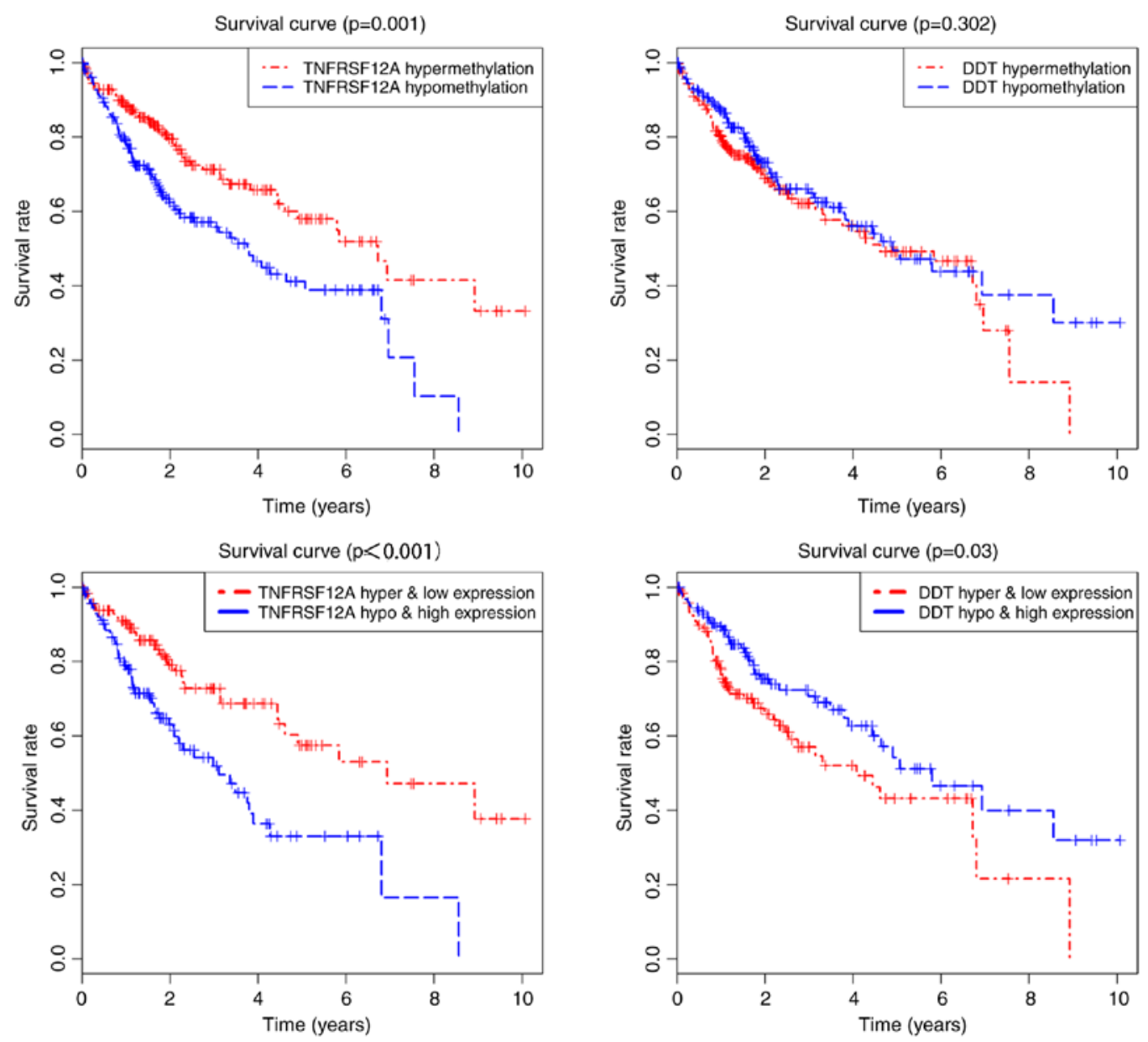

Figure 6. Survival analysis in terms of TNFRSF12A and DDT. Kaplan-Meier survival curves using the methylation status of TNFRSF12A and DDT, and survival curves plotted by combining the methylation and expression data of TNFRSF12A and DDT genes. TNFRSF12A, tumor necrosis factor receptor superfamily member 12A; DDT, D-dopachrome decarboxylase.

Multivariate Cox regression was used to generate a prognostic risk model, which was used to evaluate prognosis. The 5-year survival rate was $35.8 \%(95 \% \mathrm{CI}=27.1-47.4 \%)$ in the high-risk group and $61.7 \%(95 \% \mathrm{CI}=51.4-74.2 \%)$ in the low-risk group $(\mathrm{P}<0.0001)$. The prognostic model constructed showed a level of accuracy and sensitivity in evaluating the prognosis of patients with HCC.

The expression of TNFRSF12A is increased in various tumors, especially in HCC and breast cancer (30). As the sole signal receptor of the proinflammatory cytokine TNF superfamily member 12 , TNFRSF12A is involved in stimulating many signal transduction pathways, including the nuclear factor- $\kappa \mathrm{B}$ pathway. It has been reported that the knockout of the differentially expressed TNFRSF12A gene inhibited the proliferation and migration of HCC cells in vitro (31). High levels of TNFRSF12A expression are associated with the upregulation of matrix metallopeptidase- 9 and may be important for the progression of breast cancer; furthermore, TNFRSF12A-targeted therapy can improve survival (32). In the present study, TNFRSF12A was found to be hypomethylated in patients with $\mathrm{HCC}$ and that this was significantly correlated with prognosis. The methylation status of TNFRSF12A was negatively correlated with gene expression. The hypomethylated status of TNFRSF12A and its high expression corresponded to a significantly lower survival rate. Therefore, TNFRSF12A can be used as an independent prognostic predictor of HCC. Further analysis of the methylation loci showed that $\mathrm{cg} 00510447$ and cg26808293 were also negatively correlated with gene expression. It is, therefore, speculated that the hypomethylation of cg00510447 and cg26808293 resulted in the high expression of the TNFRSF12A gene, which may affect metabolism in HCC, and the proliferation and migration of tumor cells.

DDT (MIF2), the second member of the multi-functional proinflammatory protein macrophage mobility inhibitor superfamily, is an important mediator of the inflammation-cancer axis and plays a role in angiogenesis by inducing angiogenic factors, such as vascular endothelial growth factor and interleukin-8 (33). In lung adenocarcinoma cells, MIF and DDT have a cumulative effect on the induction of these carcinogenic factors, indicating that MIF and DDT may play a synergistic role in malignant diseases (34), however, little is known about the methylation of DDT in HCC. In the present study, it was found that DDT was hypermethylated in HCC, and that the prognosis of HCC was poorer in the hypermethylated low expression group. Further analysis indicated that cg11060661 and $\operatorname{cg} 16132339$ were also negatively correlated with gene expression. The results suggested that DDT plays a protective role in HCC and may act as a tumor suppressor gene. Further studies are required to elucidate the specific mechanisms underlying the preliminary findings of the present study. 

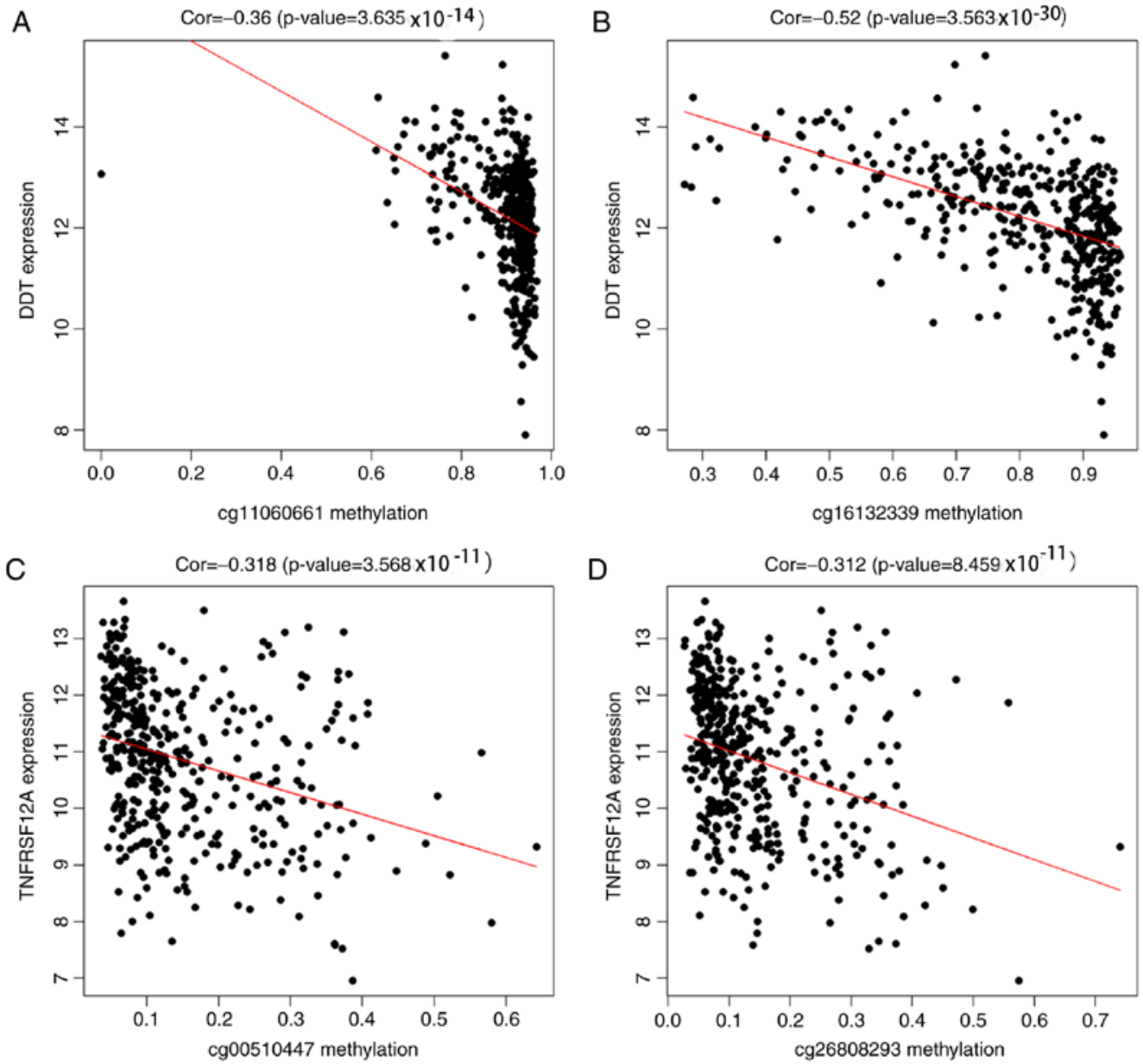

Figure 7. Correlation analysis between the methylation status of the locus and the expression level of TNFRSF12A and DDT. (A and B) Gene expression and methylation locus of DDT; (C and D) gene expression and methylation locus of TNFRSF12A. TNFRSF12A, tumor necrosis factor receptor superfamily member 12A; DDT, D-dopachrome decarboxylase; Cor, correlation.

Unlike mutations, epigenetic changes are reversible, especially DNA methylation and histone modifications (35). The expression of genes silenced by DNA methylation can be promoted in cancer cell lines using demethylating agents, such as 5-azacytidine and 5-azacytidine-2'-deoxycytidine (36), which are used in myelodysplastic syndromes and acute myeloid leukemia (37). As a novel DNA methyltransferase inhibitor, Zebularine can inhibit DNA methylation by forming a covalent complex with DNA methyltransferase 1 . The stability and half-life of Zebularine in neutral aqueous solutions is significantly better than azatadine and histamine (38). In animal experiments, Andersen et al (39) demonstrated that Zebularine response genes and demethylation signatures predictive of clinical outcome in patients with HCC, could be used as a new diagnostic and prognostic tool for selecting patients with $\mathrm{HCC}$ who may benefit from epigenetic therapy. In the target group with the highest degree of DNA CpG methylation, epigenetic therapy with Zebularine successfully inhibited tumor cell proliferation and increased apoptosis.

There have been a number of previous studies on DNA methylation in HCC. For example, the methylation of P14ARF, growth arrest and DNA damage inducible GADD45 $\beta$ and protocadherin 8 is associated with HCC $(15,40,41)$. In addition, Tao et al (42) found that seven genes (WNK2, EMILIN2, TLX3, TM6SF1, TRIM58, HIST1H4F and GRASP) were hypermethylated in HBV-related HCC and hypomethylated in paired adjacent liver tissues. Fan et al (43), using $\mid \log F C l \geq 2$ and $\mathrm{P} \leq 0.05$, identified six hub genes that were altered in HCC from The Gene Expression Omnibus; patients with high expression of mitotic arrest deficient 2-like 1, cell division cycle 20 and cyclin B1 and low expression cyclin D1, androgen receptor and estrogen receptor 1 had a shorter OS. However, these previous studies, including (15,40-43), only assessed the value of DNA methylation of single or multiple genes in HCC. Although DNA methylation determines when, where and how genes are expressed, DNA methylation at a single site does not necessarily affect the prognosis of a patient as there are multiple methylation sites on each gene (44). Only when DNA methylation affects gene expression can it affect the prognosis of a patient. Therefore, the present study combined gene expression and DNA methylation (Cor $\leq-0.3)$ from TCGA to conduct a comprehensive analysis, using this a prognostic risk model was constructed for HCC using univariate and multivariate COX regression. This model can also be used to quantify the survival time of patients with $\mathrm{HCC}$, and has strong clinical applicability. However, the present study is comprised only of bioinformatics analysis with no experimental verification. Therefore, future studies should collect cancer samples and the clinical data of patients with HCC in order to verify the accuracy of the model by detecting the DNA methylation 
status of the eight prognostic genes identified in the present study.

It can be concluded from the analysis conducted in the present study that the occurrence and development of HCC are closely related to the eight methylation-driven genes identified, including CALML3, CCNI2, TNFRSF12A, IFITM1, ENPP7P13, DDT, RASAL2-AS1 and ANKRD22. The degree of methylation and gene expression level of TNFRSF12A and DDT are significantly correlated with the prognosis of HCC and were negatively correlated with the methylation status of the following loci: $\operatorname{cg} 00510447, \operatorname{cg} 26808293, \operatorname{cg} 11060661$ and cg16132339. Experimental and clinical trials are required to further investigate the findings of the present study, which may be important for the diagnosis, treatment and prognosis of patients with HCC.

\section{Acknowledgements}

Not applicable.

\section{Funding}

The present study was supported by the National Natural Science Foundation of China (grant no. 81671946) and the Medical Scientific Research Foundation of Guangdong Province (grant no. A2015479).

\section{Availability of data and materials}

The datasets used and/or analyzed during the present study are available from the corresponding author on reasonable request.

\section{Authors' contributions}

JL conceived and designed the study. Data analysis was conducted by $\mathrm{XG}, \mathrm{JL}$ and $\mathrm{XG}$, and $\mathrm{NC}$ contributed to study methodology, software use, study administration and data validation. JL and XG wrote, reviewed and edited the manuscript. All authors read and approved the final manuscript.

\section{Ethics approval and consent to participate}

Not applicable.

\section{Patient consent for publication}

Not applicable.

\section{Competing interests}

The authors declare that they have no competing interests.

\section{References}

1. McGlynn KA, Petrick JL and London WT: Global epidemiology of hepatocellular carcinoma: An emphasis on demographic and regional variability. Clin Liver Dis 19: 223-238, 2015.

2. Torre LA, Bray F, Siegel RL, Ferlay J, Lortet-Tieulent J and Jemal A: Global cancer statistics, 2012. CA Cancer J Clin 65: 87-108, 2015

3. Shen $\mathrm{H}$ and Laird PW: Interplay between the cancer genome and epigenome. Cell 153: 38-55, 2013.
4. Kanwal R, Gupta K and Gupta S: Cancer epigenetics: An introduction. Methods Mol Biol 1238: 3-25, 2015.

5. Yang IV and Schwartz DA: Epigenetic control of gene expression in the lung. Am J Respir Crit Care Med 183: 1295-1301, 2011.

6. Widschwendter M, Jiang G, Woods C, Müller HM, Fiegl H, Goebel G, Marth C, Müller-Holzner E, Zeimet AG, Laird PW and Ehrlich M: DNA hypomethylation and ovarian cancer biology. Cancer Res 64: 4472-4480, 2004.

7. Yoder JA, Walsh CP and Bestor TH: Cytosine methylation and the ecology of intragenomic parasites. Trends Genet 13: 335-340, 1997.

8. Jones PA: Functions of DNA methylation: Islands, start sites, gene bodies and beyond. Nat Rev Genet 13: 484-492, 2012.

9. Baylin SB and Chen WY: Aberrant gene silencing in tumor progression: Implications for control of cancer. Cold Spring Harb Symp Quant Biol 70: 427-433, 2005.

10. Jones PA and Baylin SB: The epigenomics of cancer. Cell 128: 683-692, 2007.

11. Gao C, Zhuang J, Zhou C, Ma K, Zhao M, Liu C, Liu L, Li H, Feng F and Sun C: Prognostic value of aberrantly expressed methylation gene profiles in lung squamous cell carcinoma: A study based on The Cancer Genome Atlas. J Cell Physiol 234: 6519-6528, 2019

12. Feng L and Jin F: Screening of differentially methylated genes in breast cancer and risk model construction based on TCGA database. Oncol Lett 16: 6407-6416, 2018.

13. Udali S, Guarini P, Ruzzenente A, Ferrarini A, Guglielmi A, Lotto V, Tononi P, Pattini P, Moruzzi S, Campagnaro T, et al: DNA methylation and gene expression profiles show novel regulatory pathways in hepatocellular carcinoma. Clin Epigenetics 7: 43, 2015.

14. Zhong XY, Yuan XM, Xu YY, Yin M, Yan WW, Zou SW, Wei LM, Lu HJ, Wang YP and Lei QY: CARM1 methylates GAPDH to regulate glucose metabolism and is suppressed in liver cancer. Cell Rep 24: 3207-3223, 2018.

15. Zhang H, Nie W and Huang F: The correlation relationship between P14ARF gene DNA methylation and primary liver cancer. Med Sci Monit 21: 3077-3082, 2015.

16. Tomczak K, Czerwińska P and Wiznerowicz M: The Cancer Genome Atlas (TCGA): An immeasurable source of knowledge. Contemp Oncol (Pozn) 19: A68-A77, 2015.

17. Ritchie ME, Phipson B, Wu D, Hu Y, Law CW, Shi W and Smyth GK: Limma powers differential expression analyses for RNA-sequencing and microarray studies. Nucleic Acids Res 43: e47, 2015 .

18. Singh R and Mukhopadhyay K: Survival analysis in clinical trials: Basics and must know areas. Perspect Clin Res 2: 145-148, 2011.

19. Zhang CB, Zhu P, Yang P, Cai JQ, Wang ZL, Li QB Bao ZS, Zhang $\mathrm{W}$ and Jiang T: Identification of high risk anaplastic gliomas by a diagnostic and prognostic signature derived from mRNA expression profiling. Oncotarget 6: 36643-36651, 2015.

20. Chan SL, Wong VW, Qin S and Chan HL: Infection and cancer: The case of Hepatitis B. J Clin Oncol 34: 83-90, 2016.

21. Wallace MC, Preen D, Jeffrey GP and Adams LA: The evolving epidemiology of hepatocellular carcinoma: A global perspective. Expert Rev Gastroenterol Hepatol 9: 765-779, 2015.

22. Tian Y, Wong WS, Chan LY and Cheng AS: Epigenetic regulation of hepatocellular carcinoma in non-alcoholic fatty liver disease. Semin Cancer Biol 23: 471-482, 2013.

23. Kalhan SC, Edmison J, Marczewski S, Dasarathy S, Gruca LL, Bennett C, Duenas C and Lopez R: Methionine and protein metabolism in non-alcoholic steatohepatitis: Evidence for lower rate of transmethylation of methionine. Clin Sci (Lond) 121: 179-189, 2011.

24. Niculescu MD and Zeisel SH: Diet, methyl donors and DNA methylation: Interactions between dietary folate, methionine and choline. J Nutr 132 (8 Suppl): 2333S-2335S, 2002.

25. Tryndyak VP, Han T, Muskhelishvili L, Fuscoe JC, Ross SA, Beland FA and Pogribny IP: Coupling global methylation and gene expression profiles reveal key pathophysiological events in liver injury induced by a methyl-deficient diet. Mol Nutr Food Res 55: 411-418, 2011.

26. Pogribny IP, James SJ and Beland FA: Molecular alterations in hepatocarcinogenesis induced by dietary methyl deficiency. Mol Nutr Food Res 56: 116-125, 2012.

27. Bruix J, Gores GJ and Mazzaferro V: Hepatocellular carcinoma: Clinical frontiers and perspectives. Gut 63: 844-855, 2014. 
28. Jones PA and Takai D: The role of DNA methylation in mammalian epigenetics. Science 293: 1068-1070, 2001.

29. Karpf AR and Jones DA: Reactivating the expression of methylation silenced genes in human cancer. Oncogene 21: 5496-5503, 2002.

30. Wiley SR, Cassiano L, Lofton T, Davis-Smith T, Winkles JA, Lindner V, Liu H, Daniel TO, Smith CA and Fanslow WC: A novel TNF receptor family member binds TWEAK and is implicated in angiogenesis. Immunity 15: 837-846, 2001.

31. Wang T, Ma S, Qi X, Tang X, Cui D, Wang Z, Chi J, Li P and Zhai B: Knockdown of the differentially expressed gene TNFRSF12A inhibits hepatocellular carcinoma cell proliferation and migration in vitro. Mol Med Rep 15: 1172-1178, 2017.

32. Yang J, Min KW, Kim DH, Son BK, Moon KM, Wi YC, Bang SS, Oh YH, Do SI, Chae SW, et al: High TNFRSF12A level associated with MMP-9 overexpression is linked to poor prognosis in breast cancer: Gene set enrichment analysis and validation in large-scale cohorts. PLoS One 13: e0202113, 2018.

33. O'Reilly C, Doroudian M, Mawhinney L and Donnelly SC: Targeting MIF in cancer: Therapeutic strategies, current developments, and future opportunities. Med Res Rev 36: 440-460, 2016.

34. Coleman AM, Rendon BE, Zhao M, Qian MW, Bucala R, Xin D and Mitchell RA: Cooperative regulation of non-small cell lung carcinoma angiogenic potential by macrophage migration inhibitory factor and its homolog, D-dopachrome tautomerase. J Immunol 181: 2330-2337, 2008.

35. O'Brien CA, Pollett A, Gallinger S and Dick JE: A human colon cancer cell capable of initiating tumour growth in immunodeficient mice. Nature 445: 106-110, 2007.

36. Wang M, Xiao J, Shen M, Yahong Y, Tian R, Zhu F, Jiang J, Du Z, Hu J, Liu W and Qin R: Isolation and characterization of tumorigenic extrahepatic cholangiocarcinoma cells with stem cell-like properties. Int J Cancer 128: 72-81, 2011.
37. Klepin HD: Myelodysplastic syndromes and acute myeloid leukemia in the elderly. Clin Geriat Med 32: 155-173, 2016.

38. Zhou L, Cheng X, Connolly BA, Dickman MJ, Hurd PJ and Hornby DP: Zebularine: A novel DNA methylation inhibitor that forms a covalent complex with DNA methyltransferases. J Mol Biol 321: 591-599, 2002.

39. Andersen JB, Factor VM, Marquardt JU, Raggi C, Lee YH, Seo D, Conner EA and Thorgeirsson SS: An integrated genomic and epigenomic approach predicts therapeutic response to zebularine in human liver cancer. Sci Transl Med 2: 54ra77, 2010.

40. Hou XJ, Zhao QD, Jing YY, Han ZP, Yang X, Wei LX, Zheng YT, Xie F and Zhang BH: Methylation mediated Gadd $45 \beta$ enhanced the chemosensitivity of hepatocellular carcinoma by inhibiting the stemness of liver cancer cells. Cell Biosci 7: 63, 2017.

41. Cheng Z, Yunfei P, Fan Y, Qin R, Liu W and Zhang C: PCDH8 is frequently inactivated by promoter hypermethylation in liver cancer: Diagnostic and clinical significance. J Cancer 7: 446-452, 2016.

42. Tao R, Li J, Xin J, Wu J, Guo J, Zhang L, Jiang L, Zhang W, Yang $Z$ and Li L: Methylation profile of single hepatocytes derived from hepatitis B virus-related hepatocellular carcinoma. PLoS One 6: e19862, 2011.

43. Fan G, Tu Y, Chen C, Sun H, Wan C and Cai X: DNA methylation biomarkers for hepatocellular carcinoma. Cancer Cell Int 18: 140,2018

44. Zeidler R, de Freitas Soares BL, Bader A and Giri S: Molecular epigenetic targets for liver diseases: Current challenges and future prospects. Drug Discov Today 22: 1620-1636, 2017. 